\title{
Realistic model of correlated disorder and Anderson localization
}

\author{
V.V. Flambaum \\ School of Physics, University of New South Wales, Sydney 2052, Australia
}

\begin{abstract}
A conducting 1D line or 2D plane inside (or on the surface of) an insulator is considered. Impurities displace the charges inside the insulator. This results in a long-range fluctuating electric field acting on the conducting line (plane). This field can be modeled by that of randomly distributed electric dipoles. This model provides a random correlated potential with $\langle U(r) U(r+k)\rangle \propto 1 / k$. In the $1 \mathrm{D}$ case such correlations give essential corrections to the localization length but do not destroy Anderson localization.
\end{abstract}

It was recently stated in [1] that some special correlations in a random potential can produce a mobility edge (between localized and delocalized states) inside the allowed band in the 1D tight-binding model. In principle, extrapolation of this result to 2D systems may give a possible explanation of the insulator-conductor transition in dilute 2D electron systems observed in ref. [2]. In such a situation it is very important to build a reasonable model of "correlated disorder" in real systems and calculate the effects of this "real" disorder.

Usually, a $1 \mathrm{D}$ or $2 \mathrm{D}$ conductor is made inside or on the surface of an insulating material. Impurities inside the insulator displace the electric charges. However, a naive "random charge" model violates electro-neutrality and gives wrong results. Indeed, the impurities do not produce new charges, they only displace charges thus forming electric dipoles. Therefore, we consider a model of randomly distributed electric dipoles (alternatively, one can consider a spin glass model which gives the correlated random magnetic field). The dipoles have long-range electric field. Therefore, the potentials at different sites turn out to be correlated.

The potential energy produced by the system of the dipoles $d_{j}$ is equal to

$$
U(r)=e \sum_{j} \mathbf{d}_{\mathbf{j}} \nabla \frac{1}{\left|\mathbf{r}-\mathbf{R}_{\mathbf{j}}\right|} .
$$

The average value of this potential is zero if $\left\langle\mathbf{d}_{\mathbf{j}}\right\rangle=\mathbf{0}$. The fluctuations of the potential at a given site are as follows

$$
\langle U(r) U(r)\rangle=\frac{e^{2} d^{2} \rho}{3} \int \frac{d^{3} R}{R^{4}}=\frac{4 \pi e^{2} d^{2} \rho}{3 r_{0}} .
$$

Here we assumed that $\left\langle d_{i}^{\alpha} d_{j}^{\beta}\right\rangle=d^{2} / 3 \delta_{i l} \delta_{\alpha \beta}$ where $\alpha$ and $\beta$ are space indices, and the dipoles are distributed in space with a constant density $\rho$. We had to introduce a cut-off parameter $r_{0}$ since the integral diverges at small $R$. This parameter is, in fact, the geometrical size of the dipole. Indeed, inside the radius $r_{0}$ the electric field cannot be described by the dipole formula and the real potential $U(r)$ does not contain the singularity $1 / r^{2}$ which leads to the divergence of the integral. Our cut-off corresponds to a zero field inside the sphere of radius $r_{0}$ [3].

The correlator of the potentials at the points $\mathbf{r}_{1}$ and $\mathbf{r}_{2}$ is equal to

$$
\left.\left\langle U\left(\mathbf{r}_{1}\right) \mathbf{U}\left(\mathbf{r}_{\mathbf{2}}\right)\right\rangle=\mathbf{e}^{2} \sum_{\mathbf{i}, \mathbf{j}}<\mathbf{d}_{\mathbf{i}} \nabla \frac{\mathbf{1}}{\left|\mathbf{r}_{\mathbf{1}}-\mathbf{R}_{\mathbf{i}}\right|} \mathbf{d}_{\mathbf{j}} \nabla \frac{\mathbf{1}}{\left|\mathbf{r}_{\mathbf{2}}-\mathbf{R}_{\mathbf{j}}\right|}\right\rangle=\frac{\mathbf{e}^{2} \mathbf{d}^{2}}{\mathbf{3}} \sum_{\mathbf{j}}\left(\nabla \frac{\mathbf{1}}{\left|\mathbf{r}_{\mathbf{1}}-\mathbf{R}_{\mathbf{j}}\right|}\right)\left(\nabla \frac{\mathbf{1}}{\left|\mathbf{r}_{\mathbf{2}}-\mathbf{R}_{\mathbf{j}}\right|}\right) .
$$

Assume that the dipoles are distributed in space with a constant density $\rho$. Then we have

$$
\left\langle U\left(\mathbf{r}_{1}\right) \mathbf{U}\left(\mathbf{r}_{2}\right)\right\rangle=\frac{\mathbf{e}^{2} \mathbf{d}^{2} \rho}{\mathbf{3}} \int \mathbf{d}^{3} \mathbf{R}\left(\nabla \frac{\mathbf{1}}{\left|\mathbf{r}_{1}-\mathbf{R}\right|}\right)\left(\nabla \frac{\mathbf{1}}{\left|\mathbf{r}_{2}-\mathbf{R}\right|}\right)=\frac{4 \pi \mathbf{e}^{2} \mathbf{d}^{2} \rho}{3\left|\mathbf{r}_{1}-\mathbf{r}_{2}\right|} .
$$

The integral here is convergent and can be easily calculated for $r_{0}=0$ using integration by parts. It is interesting that the result is the same (does not depend on $r_{0}$ ) for any $r_{0}<\left|\mathbf{r}_{\mathbf{1}}-\mathbf{r}_{\mathbf{2}}\right| / \mathbf{2}$. Indeed, this problem is mathematically equivalent to the calculation of the electrostatic energy of two charged spheres of the radius $r_{0}$ ( the interaction energy is proportional to the cross term $2 \mathbf{E}_{\mathbf{1}} \mathbf{E}_{\mathbf{2}} / \mathbf{8} \pi$ in the energy density of electric field $\left.\mathbf{E}^{\mathbf{2}} / \mathbf{8} \pi ; \mathbf{E}_{\mathbf{1 , 2}}=\mathbf{e} \nabla\left(\mathbf{1} /\left|\mathbf{r}_{\mathbf{1 , 2}}-\mathbf{R}\right|\right)\right)$. The answer is known: the interaction energy is equal to $e^{2} /\left|\mathbf{r}_{\mathbf{1}}-\mathbf{r}_{\mathbf{2}}\right|$ if $2 r_{0}<\left|\mathbf{r}_{\mathbf{1}}-\mathbf{r}_{\mathbf{2}}\right|$.

Thus, we obtained the following result for the normalized correlator

$$
\xi(k) \equiv \frac{\langle U(\mathbf{r}) \mathbf{U}(\mathbf{r}+\mathbf{k})\rangle}{\left\langle U^{2}\right\rangle}=\frac{r_{0}}{k} .
$$


We see that the correlations in the dipole random potential decay inversely proportional to the distance.

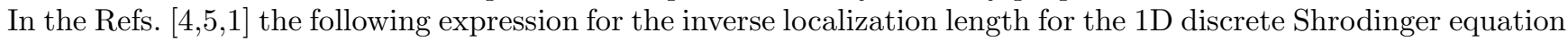

$$
\psi_{n+1}+\psi_{n-1}=\left(E+\epsilon_{n}\right) \psi_{n}
$$

has been obtained:

$$
\begin{gathered}
l^{-1}=\frac{\epsilon_{0}^{2} \phi(\mu)}{8 \sin ^{2} \mu} ; \\
\phi(\mu)=1+2 \sum_{k=1}^{\infty} \xi(k) \cos (2 \mu k)
\end{gathered}
$$

Here the eigenenergy is $E=2 \cos \mu, \epsilon_{n}=U\left(r_{n}\right), \epsilon_{0}^{2}=\left\langle U^{2}\right\rangle$. This equation has been derived in the approximation $\epsilon_{n} \ll 1$. Now we can substitute the correlator $\xi(k)=r_{0} / k$ into this equation. The result is

$$
\phi(\mu)=1-2 r_{0} \ln |2 \sin \mu| .
$$

In this equation $r_{0}$ is measured in units of the lattice constant. The minimum of $\phi$ is given by $\phi_{\min } \simeq 1-1.4 r_{0}$. The delocalization corresponds to $\phi=0\left(r_{0}=0.72\right)$. This condition seems to be impossible to satisfy. Indeed, the equation $\xi(k)=r_{0} / k$ is valid for $r_{0}<0.5$. The realistic value of $r_{0}$ is smaller than this limit. A typical dipole size in molecules is about one Bohr radius while the lattice constant is about five times larger. This gives an estimate $r_{0} \sim 0.2$. Also, any short-range fluctuations increase $\left\langle U^{2}\right\rangle$ and reduce the normalized long-range correlator $\xi(k)$, see eq. (5).

However, the correlations change the localization length significantly. It is well known that in the $2 \mathrm{D}$ case the localization length is very sensitive to parameters of the problem. It would be a natural guess that in the $2 \mathrm{D}$ case the correlations (5) due to the long- range character of the dipole field are more important than in the 1D case, and that they may lead to delocalization.

Acknowledgments. The author acknowledges the support from Australian Research Council. He is grateful to F. Izrailev and A. Krokhin for discussions and to V.Zelevinsky for valuable comments and hospitality during the stay in MSU Cyclotron laboratory when this work was done.

[1] F.M. Izrailev and A.A. Krokhin. Preprint cond-mat 9812209.

[2] S.V. Kravchenko et al., Phys. Rev. Lett. 77 ,4938 (1996).

[3] The electric field equal to the dipole field outside the sphere and zero field inside corresponds to a "spherical dipole" electric dipole consisting of two oppositely charged spheres with a small distance between their centers.

[4] M.Griniasty and S. Fishman, Phys. Rev. Lett. 60, 1334 (1988).

[5] J.M. Luck, Phys. Rev. B 39, 5834 (1989). 